\title{
Summary Abstract: Surface core excitons in III-V semiconductors
}

\author{
M. S. Daw, D. L. Smith, and T. C. McGill \\ California Institute of Technology, Pasadena, California 91125
}

(Received 18 March 1981; accepted 18 June 1981)

PACS numbers: 71.35. $+\mathrm{z}, 73.20 . \mathrm{Cw}, 72.80 . \mathrm{Ey}$
Recent experiments have shown that the cation core excitons on the (110) surface of many III-V semiconductors have very large binding energies. ${ }^{1}$ They are sometimes reported to be bound by as much as $\gtrsim 0.8 \mathrm{eV}$, tightly bound compared to bulk binding energies of $\$ 0.1 \mathrm{eV}$. To explore this phenomenon, we have calculated the binding energies and oscillator strengths of core excitons on the (110) surface of $\mathrm{GaAs}, \mathrm{GaSb}$, $\mathrm{GaP}$, and InP.

At last year's PCSI conference, Wang and Joannopolous ${ }^{2}$ presented their tight-binding calculation of $\mathrm{Ga}$ core excitons on the unrelaxed (110) GaAs surface. This treatment used an unrealistic exciton potential and did not allow for surface relaxation which is known to push empty Ga derived surface states from the band gap into the band. ${ }^{3}$ With an inherently surface related excitation, it is important that one include surface relaxation so that the final state for the electron is described accurately.

We present here a theoretical picture of anion and cation core excitons for III-V surfaces, using relaxed surface geometries. A qualitative picture serves to summarize our concept of surface core excitons. On the (110) surface of IIIV's there are two species of dangling bonds: the cation dangling bond, which because of surface relaxation is mostly $p$ $(3 \% s)$ and is completely empty, and the anion dangling bond which is mostly $s p^{3}$-hybrid and fully occupied. A dipole transition out of a cation $d$ - or $s$-core level, for example, goes easily to the empty, $p$-type dangling bond on that atom. The electron in this final state is localized near the hole and the Coulombic attraction produces strong binding. The $d$ - or $s$ core excitation would have different transition energies, but closely similar binding energies because the potential due to a $s$-core hole is hardly different from that due to a $d$-core hole, as viewed by the excited electron.

An excitation out of a surface cation $p$-core level would have the same binding energy as $s$ - or $d$-core excitons, but this transition couples to the small $s$-component of the dangling bond, reducing the oscillator strength by a factor of about 30 from the cation $d$-core level transition.

In the case of excitation out of the anion core, the on-site dangling bond is fully occupied and the electron must go to other unoccupied states, any of which are less localized. One would therefore expect less binding for surface anion core excitons than for the cation excitation. We thus find a sharp contrast between anion and cation surface core excitations.

The presence of the dangling bonds causes surface core excitons to be markedly different from bulk ${ }^{4}$ or interface ${ }^{5}$ core excitons. The dangling bond state allows the localization of the electron not possible in bulk, producing the deeper binding.

The quantitative calculations were based on a realistic tight-binding Hamiltonian for relaxed surface geometries. We employed a Koster-Slater approach, very similar to our previous surface vacancy calculations. ${ }^{3}$ The effective on-site potential is viewed as a shift in the atomic state energies of the excited surface atom, which is estimated from atomic calculations. Specifically, in the case of a Ga core excitation, for example, the potential is described by shifting the atomic levels on the Ga atom to the energy levels of a Ge atom. This reasonably assumes that the core hole resembles a positive charge added to the nucleus of the excited atom. The potential can be considered as effectively localized to one atom because in this region the potential goes as $-e^{2} / r$, whereas for larger distances the screening by the rest of the solid dampens the potential to $-e^{2} / \epsilon r$, where $\epsilon=12$ for GaAs, for example. In the bulk, the long-range part of the potential is important. For surface excitations, the binding energy is strongly determined by the short-range part. ${ }^{6}$

In Table I we present our results for binding energies and oscillator strengths. The cation $p$ and $d$ core excitations on $\mathrm{GaAs}, \mathrm{GaP}$, and InP are seen to be strongly bound. The $\mathrm{Ga}$ core excitations on $\mathrm{GaSb}$ are weakly bound with respect to the conduction band minimum. We find no binding for any of the anion core excitations; we find that these states occur in the conduction band. We also present the oscillator strengths relative to the $d$-core exciton for deeply bound excitons. In $\mathrm{GaAs}, \mathrm{GaP}$, and InP, we see that the cation $p$-core transition is more than an order of magnitude weaker than the cation $d$-core, though they have the same binding. This difference in magnitude reflects the small $s$-character of the cation dangling bond.

Experimental values for the binding energies of the excitations in the table are somewhat scattered. The values for $\mathrm{Ga}$ $(3 d)$ binding on $\mathrm{GaSb}$ vary by about $0.6 \mathrm{eV}$, for example. ${ }^{7}$ For this reason, we defer a detailed discussion and comparison with experiment to a longer publication. ${ }^{6}$ In general, though, the calculated binding energies are in agreement with much of the reported data.

Also, experimentally, the cation $p$-core excitons have not appeared, while the cation $d$-core excitons have been the ones to be reported; this is consistent with our evaluation of oscillator strengths.

Anion core excitons have not been reported except for a rather incomplete description by Bauer of an observed As $(3 p)$ exciton on GaAs with binding on the order of the band gap. 
TABLE I.

\begin{tabular}{clcc}
\hline \hline & & \multicolumn{2}{c}{ Theory } \\
Material & Core & $\begin{array}{c}\text { Binding } \\
\text { energy } \\
\text { level }\end{array}$ & $\begin{array}{c}\text { Relative } \\
\text { oscillator } \\
\text { strength } \\
(d=100 \%)\end{array}$ \\
\hline \multirow{6}{*}{$\mathrm{GaAs}$} & $\mathrm{Ga}(3 p)$ & 0.9 & $3 \%$ \\
& $\mathrm{Ga}(3 d)$ & 0.9 & $100 \%$ \\
& $\mathrm{As}(3 p)$ & $\geq 0.0$ & $\ldots$ \\
$\mathrm{GaP}$ & $\mathrm{As}(3 d)$ & $\geq 0.0$ & $\ldots$ \\
& $\mathrm{Ga}(3 p)$ & 0.8 & $3 \%$ \\
& $\mathrm{Ga}(3 d)$ & 0.8 & $100 \%$ \\
& $\mathrm{P}(2 p)$ & $\leq 0.0$ & $\ldots$ \\
$\mathrm{GaSb}$ & $\mathrm{P}(2 d)$ & $\leq 0.0$ & $\ldots$ \\
& $\mathrm{Ga}(3 p)$ & $\leq 0.0$ & $\ldots$ \\
& $\mathrm{Ga}(3 d)$ & $\leq 0.0$ & $\ldots$ \\
& $\mathrm{Sb}(4 p)$ & $\leq 0.0$ & $3 \%$ \\
$\mathrm{InP}$ & $\mathrm{Sb}(4 d)$ & $\leq 0.0$ & $100 \%$ \\
& $\mathrm{In}(4 p)$ & 0.6 & \\
\hline \hline & $\mathrm{In}(4 d)$ & 0.6 & \\
& $\mathrm{P}(2 p)$ & $\leq 0.0$ & \\
& $\mathrm{P}(2 d)$ & $\leq 0.0$ & \\
\hline
\end{tabular}

This observation contradicts our picture of surface core excitons. For a surface $\mathrm{As}(3 p)$ electron to be promoted to a dangling bond, it must wind up principally on the neighboring surface $\mathrm{Ga}$ dangling bonds, and the resulting separation between hole and electron is larger than in the case of a Ga core exciton where the electron can reside on the same atom as the hole. The resulting larger separation for the As excitation lowers the Coulombic interaction and, one would expect, also the binding energy. One would believe that the As exciton would have less binding, not more, than the Ga exciton. Also, the selection rules valid for the cation excitation would not hold for the anion case because the latter transition involves states centered on separate sites. The reported As $(3 p)$ exciton is clearly in contradiction with this theory. Preliminary results from other experiments do not confirm Bauer's observation. ${ }^{8}$

Our calculations are confirmed somewhat by calculations by Swarts et al. ${ }^{9}$ in this volume. They calculate for clusters of surface atoms the transition energies of various core excitations and the nature of the electron's final state. The anion excitation is less bound, in particular, than the cation exciton, in agreement with our results.

We have presented a theoretical picture of anion and cation surface core excitons for III-V semiconductors. Cation $d$-core excitons are seen to generally have large binding with the cation $p$-core excitons having the same binding but lower oscillator strength. Anion core excitations are not seen to be bound.

${ }^{1}$ R. S. Bauer, R. Z. Bachrach, S. A. Flodstrom, and J. C. McMenamin, J. Vac. Sci. Technol. 14, 378 (1977). J. van Laar, A. Huijser, and T. L. van Rooy, J. Vac. Sci. Technol. 14, 894 (1977). W. Gudat and D. E. Eastman, J. Vac. Sci. Technol. 13, 831 (1976). D. E. Eastman, T. C. Chiang, P. Heimann, and F. J. Himpsel, Phys. Rev. Lett. 45, 656 (1980).

${ }^{2}$ Y. Wang and J. D. Joannopoulos, J. Vac. Sci. Technol. 17, 997 (1980).

${ }^{3}$ M. S. Daw and D. L. Smith, Phys. Rev. B 20, 5150 (1979).

${ }^{4}$ S. M. Kelso, D. E. Aspnes, C. G. Olson, D. W. Lynch, and D. Finn, Phys. Rev. Lett. 45, 1032 (1980).

${ }^{5}$ H. P. Hjalmarson, R. E. Allen, H. Büttner, and J. D. Dow, J. Vac. Sci. Technol. 17, 993 (1980).

${ }^{6}$ M. S. Daw, D. L. Smith, and T. C. McGill (to be published).

${ }^{7}$ The last three papers of Ref. 1 list $\mathrm{Ga}(3 d)$ binding energies on $(110) \mathrm{GaSb}$ as $0.1 \mathrm{eV}, \approx 0.1 \mathrm{eV}$, and $\gtrsim 0.7 \mathrm{eV}$, respectively.

${ }^{8} \mathrm{C}$. A. Swarts, W. A. Goddard, III and T. C. McGill, these proceedings.

${ }^{9} \mathrm{P}$. Zürcher (private communication). 